\title{
Septal hyperreactivity: A multivariate analysis of neuroanatomical correlates
}

\author{
FRED H. GAGE \\ Chemistry of Behavior Program, Texas Christian University, Fort Worth, Texas 76129 \\ and \\ DAVID S. OLTON and GREGORY L. MURPHY \\ The Johns Hopkins University, Baltimore, Maryland 21218
}

\begin{abstract}
A multivariate data analysis was used to obtain a quantitative comparison of the behavioral syndromes following different lesions, all of which have been described as producing hyperreactivity. Male rats were tested preoperatively and after radio frequency lesions in the septum, medial preoptic area, total preoptic area, medial olfactory stria, and ventromedial hypothalamus. Lesions of the total preoptic area produced a hyperreactivity similar to that found after septal lesions, while lesions of the medial preoptic and the medial olfactory stria were generally ineffective. Destruction of the ventromedial hypothalamus produced a slight hyperreactivity, but not as great as that following septal lesions. The results are discussed in terms of a common neuroanatomical and/or biochemical pathway mediating the different types of reactivity.
\end{abstract}

Overresponsiveness to environmental stimuli can . follow a number of different manipulations, including isolation (Valzelli \& Garattini, 1972), drugs (Nakamura \& Thoenen, 1972), shock (Eichelman, 1971), and lesions (Brady \& Nauta, 1953). One of the best documented syndromes of overresponsiveness is that following septal lesions. Rats with large septal lesions that include the lateral septum demonstrate a hyperactivity to somatosensory stimuli and an exaggerated freezing response in a novel environment (see Gage \& Olton, 1975; Olton \& Gage, 1974 , for reviews), an increased aversion to brightly illuminated areas (see Jenko, Gage, \& Olton, 1976, for a review), and an increase in water consumption (Blass \& Hanson, 1970). All these behavioral changes occur within $24 \mathrm{~h}$ following surgery. Over time, the hyperreactivity to somatosensory stimuli and the exaggerated freezing response in a novel environment gradually diminish to normal levels, but the increased aversion to brightly illuminated areas appears to be permanent.

Hyperreactivity has been reported after damage to other brain structures, including the area ventral to the septum around the anterior preoptic area (Albert \& Richmond, 1976), the olfactory bulbs (Malick, 1972), and raise the possibility that all of the above lesions may produce similar behavioral

\footnotetext{
We thank Selby Evans for his help in the use of the APL data analysis system and TCU/Research Foundation for funding during the preparation of the manuscript. Send reprint requests and correspondence to: Fred H. Gage, Chemistry of Behavior Program, Department of Psychology, Texas Christian University, Fort Worth, Texas 76129.
}

syndromes, suggesting a common neuroanatomical or biochemical mechanism underlying them. Unfortunately, these studies differ in a variety of ways, such as the sex of the animal, the means of producing the lesion, and the behavioral tests used, so that the necessary quantitative comparisons cannot be carried out. To overcome this difficulty and evaluate the hypothesis that the hyperreactive syndrome observed after each of these lesions is the same, the present study used the same sex of rats (males), the same lesion procedures (radio-frequency current), and the same battery of behavioral tests to compare the performance of rats with lesions in the septum, preoptic area, ventromedial hypothalamus, and olfactory bulbs.

\section{Subjects}

\section{METHODS}

The subjects were 48 male Sprague-Dawley rats weighing between 250 and $300 \mathrm{~g}$ at the start of testing. All rats were housed individually with ad-lib food and water and maintained on a 12-h-light/12-h-dark cycle.

\section{General}

All rats were handled for 2 days prior to the initiation of testing. They were tested for 2 consecutive days preoperatively. Following surgery, the cages containing the rats were given numbers that were different from those they had had preoperatively, so the experimenter did not know either the preoperative scores of the rats or the intended surgery at the time of postoperative testing. Postoperatively, all rats were tested on Days 1, 6, 16, and 21.

\section{Body Weight}

The rats were provided with ad-lib Purina Lab Chow in their home cages throughout testing. Body weights were recorded at the same time each day. 


\section{Water Intake}

Rats were provided with ad-lib water in their home cages throughout testing. Water intake was recorded at the same time each day by weighing the water bottle attached to the cage.

\section{Novel Environment}

Apparatus. The open field was a wooden box, $75 \mathrm{~cm}$ square, with walls that were $45 \mathrm{~cm}$ high. The floor was divided into $15 \times 15 \mathrm{~cm}$ squares by white lines drawn on the floor. A startbox, $15 \times 15 \times 15 \mathrm{~cm}$, was connected to the open field by a guillotine door.

Procedure. Each subject was tested once on each test day. He was placed in the startbox, and the guillotine door to the open field was opened. Five minutes were allowed for the rat to enter the open field. If he had not left the startbox at the end of this time, he was gently pushed out into the open field. When the rat entered the open field, the guillotine door to the startbox was closed. The rat was allowed to explore freely for 2 min and then removed. Half the animals were tested at the beginning of the light cycle and half of the animals were tested at the end of the light cycle.

Behavioral measures. "Emergence time" was the number of seconds required for the rat to leave the startbox and enter the open field. If he had not done so by the end of $5 \mathrm{~min}$, a score of $300 \mathrm{sec}$ was given. "Lines crossed" was the number of lines in the open field crossed by the rat. A crossing was defined as both rear legs crossing over a line in the open-field box. "Rears" was the number of times the animal lifted both feet off the floor simultaneously and reared up on his hindlegs.

\section{Reactivity}

Apparatus. The apparatus was a tower, $90 \mathrm{~cm}$ high and $30 \mathrm{~cm}$ square. Three sides were of hardware cloth, the fourth of Plexiglas. The Plexiglas side was cut out at the bottom so that a rat cage could be slipped into the tower.

Procedure. Each rat was given one test on each test day. The rat was carried in his home cage to the tower. Four different stimuli were presented, and the responses to these stimuli scored into three or four categories, with a score of 0 reflecting the weakest response in all cases. Each stimulus was presented to each rat several times: if the responses to the presentations varied, the weakest response was recorded. Following testing, the rat was returned to his home cage.

Behavioral measures. The four stimuli, the patterns of response to each of them, and the scores given the various patterns of responses are summarized in Table 1.

\section{Surgery}

Each rat was anesthetized with Equithesin (Jensen-Salsbury), $2.2 \mathrm{cc} / \mathrm{kg}$, given $.25 \mathrm{ml}$ of atropine, and placed in a stereotaxic instrument. The scalp was incised and retracted, and holes were drilled through the skull in the appropriate locations. The eight control rats were then removed from the stereotaxic apparatus, and their scalps sutured, while the other rats had a stainless steel electrode lowered a few millimeters into neocortex before having their scalps sutured. Rats receiving lesions had radio frequency current from a Grass LM-4 passed through a stainless steel electrode, insulated except for the tip, which was lowered into the appropriate location in the brain. The scalp was sutured, and the rat removed from the stereotaxic instrument. Lesions were intended for the septum, medial olfactory stria (MOS), medial preoptic area (MPOA), the total preoptic area (TPOA), and the ventromedial nucleus of the hypothalamus (VMN).

\section{Histology}

Each rat was anesthetized with ether and perfused with $0.9 \%$ saline followed by Heidenhain's solution without mercuric chloride. The brain was dehydrated, cleared in cedarwood oil, and embedded in paraffin. Sections, $10 \mu \mathrm{m}$ thick, were taken
Table 1

\begin{tabular}{ll}
\hline \multicolumn{1}{c}{ Stimulus } & \multicolumn{1}{c}{ Score } \\
\hline 0: & no response; slight orienting to \\
stimulus
\end{tabular}

throughout the lesion area. Every tenth section was mounted on a slide stained with luxol fast blue for myelinated fibers and cresyl violet for cell bodies. Each lesion was reconstructed on appropriate diagrams of a rat brain atlas by König and Klippel (1963), and areas of cell loss, degeneraton, and gliosis were noted.

\section{Data Analysis}

For each behavioral measure, for each rat, the mean score on the 2 preoperative test days was subtracted from the score obtained on each postoperative test. Thus, all scores are difference scores reflecting the change in performance for an individual rat as a result of surgery.

The behaviors measured across all groups were analyzed by a discriminant function analysis. Two groups of variables (behaviors) were found to be highly correlated, and thus were combined into single measures. Specifically: (1) The four measures of reactivity to somatosensory stimulation were found to be highly intercorrelated by evaluating scores in this study as well as by evaluating these same measurements in other studies (Gage \& Olton, 1975). This combination of four measures will be referred to as reactivity scores in the future. (2) A principal components analysis was performed on the remaining variables and the correlations of the first principal component with these variables were as follows: reactivity, .24; lines crossed, .88; latency to emerge, - .63; rears, .69 ; weight gain, - .39; and water consumed, .54. Lines crossed, rears, and latency-to-emerge scores were standardized and combined according to sign because of their high correlation with the first principal component. The first principal component accounted for $36 \%$ of the variance, while the second accounted for only $20 \%$. This combination of measures will be referred to as novel environment. 


\section{RESULTS}

\section{Preoperative Behavior}

The behavior of all rats during preoperative testing was homogeneous. In the novel environment test, the rats were active, leaving the startbox quickly and exploring the open field. In the reactivity test, the rats showed little or no reaction to the various stimuli. The water intake and food intake were stable for 5 days prior to a lesion.

\section{Histology}

Nine animals were discarded from behavioral analysis because lesions were misplaced or asymmetrical. The remaining animals were divided into groups on the basis of lesion location. For each group of rats, the histological data are summarized in Figure 1, which presents the maximum and minimum extent of cell loss at the level of greatest destruction. Septal lesions $(N=5)$ were large, destroying medial and lateral septal nuclei, but sparing nonseptal structures. MOS lesions $(\mathrm{N}=5)$ destroyed both the stria and the medial portions of the olfactory tubercle. MPOA lesions $(\mathrm{N}=6)$ were ventral to the anterior commissure and anterior to the anterior hypothalamic area. They included primarily the vertical branch of the diagonal band in the medial
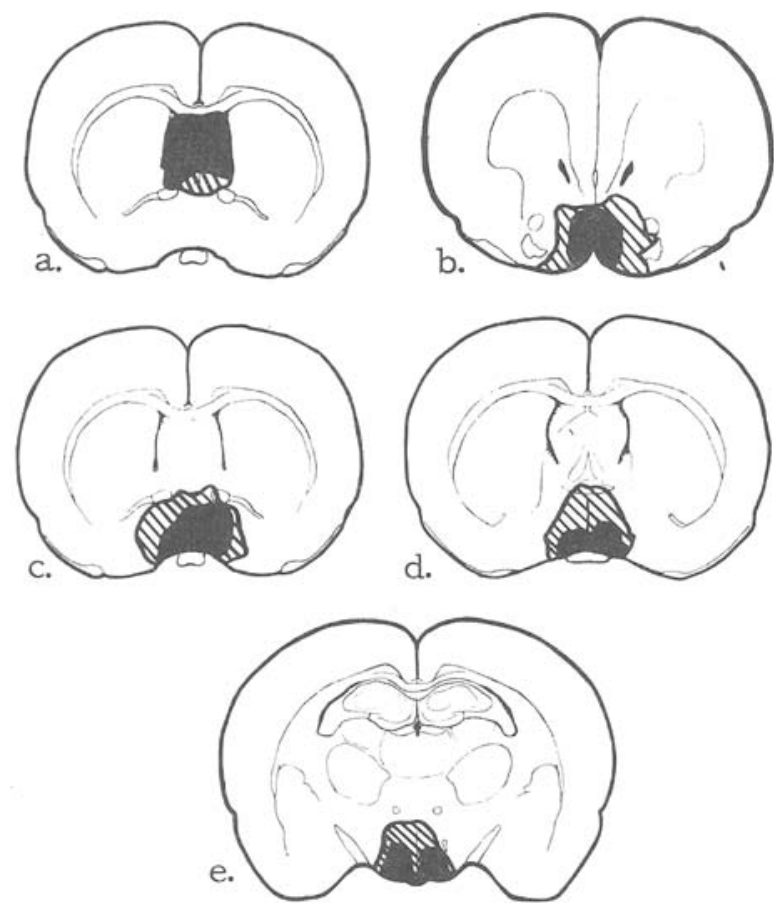

Figure 1. Reconstruction of largest lesion (diagonal hatching) and smallest lesion (solid) for rats with lesions of: (a) medial olfactory stria; (b) septal; (c) total diagonal band of Broca; (d) medial diagonal band of Broca; (e) ventromedial hypothalamus.
Table 2

Weights Attributed to Behaviors and Percent Accounted for by the First Discriminant Function on Postoperative Days 1 and 16

\begin{tabular}{lrr}
\hline & \multicolumn{2}{c}{ Weights } \\
\cline { 2 - 3 } & Day 1 & Day 16 \\
\hline Body Weights & .21 & 1.49 \\
Water Intake & .39 & -.75 \\
Novel Environment & .14 & 1.45 \\
Reactivity & -1.30 & 1.21 \\
Percent Variance & .73 & .42 \\
\hline
\end{tabular}

preoptic area. TPOA lesions $(\mathrm{N}=6)$ included the area destroyed by the medial lesion described above, plus the lateral portions of the preoptic area and the horizontal branches of the diagonal band. VMN lesions $(N=10)$ all destroyed some part of the ventromedial nucleus, and often included damage to the anterior hypothalamic nucleus, dorsomedial hypothalamic nucleus, or lateral hypothalamic area as well.

\section{Postoperative Behavior}

Rats in the control group performed essentially the same postoperatively as they had preoperatively and there was little change in their scores. Septal lesions produced the expected behavioral changes. In the reactivity test, rats with septal lesions received higher scores on all measures, reflecting the hyperreactivity commonly described following septal lesions. In the novel environment, rats with septal lesions were reluctant to leave the startbox, and when in the open field tended to freeze; thus, emergence time increased, while lines crossed and rears decreased from preoperative levels. Body weight remained about the same and water intake increased slightly.

Two critical items are revealed by a discriminant function analysis: (1) the weights (for standardized variables), which are the relative contribution of each behavior to the discriminant function, and (2) the proportion of the variance accounted for by the discriminant function. Table 2 presents these items for the data obtained Day 1 after surgery. These data clearly show that (1) the reactivity score is the most important, i.e., has the highest weighted value on the first discriminant function, and (2) the group differences account for $73 \%$ of the total variance on the first discriminant function. Thus, the first discriminant function, which provides the optimal discrimination between groups, reveals hyperreactivity as the measure that maximally defines it. The weights for the first discriminant function were used to compute a score for each rat. The values give the combined weighted scores of variables for each group which best discriminates between the groups. The results are presented in Table 3. 
Table 3

Mean and Standard Deviation of the Computed Weights Assigned Each Behavior on the First Discriminant Function Multiplied by the Standardized Deviation Score on Postoperative Day 1

\begin{tabular}{lcc}
\hline & Mean & SD \\
\hline Control & .12 & .05 \\
Septal & $-.29^{*}$ & .12 \\
TPOA & $-.25^{*}$ & .14 \\
VMN & $-.04^{*} \dagger$ & .15 \\
MPOA & .12 & .05 \\
Olfactory & .27 & .05 \\
\hline
\end{tabular}

* Significantly different from controls, $p<.05$.

tSignificantly different from septal and TPOA groups, $p<.05$.

The septal and TPOA groups are different from the control, the MOS, and the MPOA groups. The animals with VMN fall between the two groups, with a leaning toward the hyperreactive groups.

On Day 16, when the reactivity had subsided for the most part, the other behaviors contributed more to the distinction between the groups, and as a result, the differentiation between the groups was considerably less, as demonstrated by the low $42 \%$ of the variance accounted for in the first discriminant function. The results are summarized in Table 2 .

\section{DISCUSSION}

Behavioral measures of body weight, water intake, reactivity to somatosensory stimuli, and reaction to a novel environment were obtained for rats preoperatively and after lesions. Lesions were placed in the septum, to establish the well-documented syndrome of hyperreactivity, and in other areas of the brain that have been described as producing a similar type of hyperreactivity-the preoptic area, the medial olfactory stria, and the ventromedial hypothalamus. A multivariate analysis of the data was carried out to obtain a quantitative comparison between the behavioral syndrome elicited by the septal lesions and the behavioral syndromes elicited by the other lesions.

On the first postoperative test day, the first discriminant function accounted for $73 \%$ of the variance, indicating that it was a strong means of distinguishing among the groups. On this function, the most discriminating behavior was the reactivity score. Quantitative analysis indicated that the rats with lesions of the TPOA behaved almost identically to rats with septal lesions, and that rats with lesions of the MOS or the MPOA were similar to controls. Rats with VMN lesions ranked between controls and those with septal lesions. The results suggest that there were three discrete groups of rats, as defined by their behavior on the first postoperative day: very reactive ones (septal and TPOA), slightly reactive ones (VMN), and unreactive ones (controls, MOS, and MPOA).

The lack of reactivity following lesions restricted to the MOS is consistent with the results of Cain (1974), who demonstrated that reactivity following olfactory bulb lesions is dependent on the amount of tissue caudal to the olfactory bulbs that is included in the lesion. These data are consistent in pointing to an area in the septum or the diagonal band region that is critical for eliciting increased reactivity. The slight increase in reactivity after VMN lesions is consistent with Singh's (1969) results which demonstrated a clear difference in the type of reactivity following lesions of the septum and lesions of the VMN. Variables such as the sex of the animal and the manner in which the lesion is produced may influence the magnitude of hyperreactivity observed following ventromedial hypothalamic lesions (Reynolds, 1963a, 1963b, 1965a, $1965 \mathrm{~b})$, out the present study demonstrates that when these variables are held constant, the hyperreactive syndrome resulting from septal lesions is markedly different from that following VMN lesions.

The septum provides a unique point of convergence for several different anatomic and biochemical systems prior to entry into the hippocampus or cortex. In addition, the septum provides a unique point of convergence for several descending anatomical systems, prior to subcortical innervation.

The fact that the hyperreactivity recovers suggests that the area involved in the elaboration of the hyperreactivity is at some point distant from the lesions, since no tissue regrowth is expected or observed in the destroyed septal region. Diaschisis, collateral sprouting, or denervation supersensitivity are mechanisms of recovery to explore in this context. The mechanisms are particularly relevant in light of data suggesting cortical (Yutzey, Meyer, \& Meyer, 1967), hippocampal (Gage \& Olton, 1975), and the area anterior and ventral to the septal area involvement with the septal-lesion-induced hyperreactivity (Albert \& Richmond, 1976.)

\section{REFERENCES}

Albert, D. J., \& Richmond, S. E. Neural pathway mediating septal hyperreactivity. Physiology \& Behavior, 1976, 15, 339-347.

Blass. E. M., \& Hanson, D. G. Primary hyperdipsia in the rat following septal lesions. Journal of Comparative and Physiological Psychology, 1970, 70, 87-93.

BRADY, J. V., \& NAUTA, W. J. H. Subcortical mechanisms in emotional behavior. Journal of Comparative and Physiological Psychology, 1953, 46, 339-346.

CAIN, D. P. Olfactory bulbectomy: Neural structures involved in irritability and aggression in the male rat. Journal of Comparative and Physiological Psychology, 1974, 86, 213-220.

Eichelman, B. S. Effects of subcortical lesions on shockinduced aggression. Journal of Comparative and Physiological Psychology, 1971, 74. 331-339. 
Gage, F. H., \& Olton, D. S. Hippocampal influences on hyperreactivity induced by septal lesions. Brain Research, 1975, 98, 311-325.

Jenko, P. J., Gage, F. H., \& Olton, D. S. Time-dependent fornix influence on increased light aversion induced by septal lesions. Physiology \& Behavior, 1976, 17, 607-612.

König, J. F. R., \& KLIPPEL, R. A. The rat brain: A stereotaxic atlas of the forebrain and lower parts of the brainstem. Baltimore: Williams and Wilkens, 1953.

Malick, J. B. A behavioral comparison of three lesioninduced models of aggression in the rat. Journal of Comparative and Physiological Psychology, 1972, 79, 37-45.

Nakamura, K., \& Thoenen, H. Increased irritability: A permanent behavior change induced in the rat by intraventricular administration of 6-OHDA. Psychopharmacologia, 1972, 24, 359-372.

Olton, D. S., \& Gage, F. H. Role of fornix in septal syndrome. Physiology \& Behavior, 1974, 13, 269-279.

Overall, J. E., \& Klett, L. J. Applied multivariate analysis. New York: McGraw-Hill, 1972.

REYNOLDS, R. W. Radio frequency lesions in the ventrolateral hypothalamic "feeding center." Journal of Comparative and Physiological Psychology, 1963, 56, 965-967. (a)
REYNOLDS. R. W. Ventromedial hypothalamic lesions without hyperphagia. American Journal of Physiology, 1963, 204, 60-62. (b)

ReYNolds, R. W. An irritative hypothesis concerning the hypothalamic regulation of food intake. Psychological Review, 1965, 72. 105-116. (a)

REYNOLDS, R. W. Hypothalamic lesions and disinhibition of feeding. Science, 1965, 156, 1322. (b)

SINGH, D. Comparison of hyperemotionality caused by lesions in the septal and ventral hypothalamic areas in the rat. Psychonomic Science, 1969, 16, 1.

Valzelli, L., \& Garattini, S. Biochemical and behavioral changes induced by isolation in rats. Neuropharmacology, 1972, 11, 17-22.

Yutzey, D. A., Meyer, D. R., \& Meyer, P. M. Effects of simultaneous septal and neo- or limbic cortical lesions upon emotionality in the rat. Brain Research, 1967, 5, 452-458.

(Received for publication January 12, 1978; revision accepted June 13, 1978.) 